\title{
Transverse $\Lambda$ polarization at high energy colliders
}

\author{
Daniël Boer* \\ Theory Group, KVI, University of Groningen \\ Zernikelaan 25, NL-9747 AA Groningen, The Netherlands \\ E-mail: D.Boererug.nl
}

\begin{abstract}
Measurements of transverse polarization of $\Lambda$ hyperons produced in high energy $p p$ collisions may help to address several open issues about $\Lambda$ production and polarization mechanisms, such as the amount of $S U(3)$ breaking, the importance of gluons and sea quarks, and the origin of spontaneous $\Lambda$ polarization. The process $p+p \rightarrow \Lambda^{\uparrow}+$ jet $+X$ at midrapidity is ideally suited for this purpose, for instance at LHC's ALICE experiment. New expressions and predictions are presented for the transverse $\Lambda$ polarization in this process, within a factorized description which involves transverse momentum and spin dependence in the fragmentation process. Uncertainties from the unpolarized $\Lambda$ fragmentation functions, due to the unknown magnitude of $S U$ (3) breaking and the apparent inconsistency between $p p$ and $e^{+} e^{-}$data, are investigated.
\end{abstract}

XVIII International Workshop on Deep-Inelastic Scattering and Related Subjects, DIS 2010 April 19-23, 2010

Firenze, Italy

*Speaker. 


\section{Introduction}

It is well-known since the mid-1970's that $\Lambda$ hyperons produced in unpolarized $p p$ collisions are to a large degree polarized transversely to the production plane [1]. There have been many experimental and theoretical investigations aimed at understanding this striking polarization phenomenon, but no consensus has been reached about its origin. One of the difficulties in interpreting the available (mostly fixed target) data is that they are not or only partly in a region where a factorized description of the cross section is expected to be applicable. For a comprehensive review of these relatively low energy data $(\sqrt{s} \leq 62 \mathrm{GeV})$ see Ref. [2]. New high-energy hadron collider data would be very welcome, for instance from RHIC, Tevatron, or LHC, but there the capabilities to measure the $\Lambda$ polarization $P_{\Lambda}$ via the self-analyzing parity violating decay $\Lambda \rightarrow p \pi^{-}$are typically restricted to the midrapidity region $(\eta \approx 0)$, where protons can be identified ${ }^{1}$. For symmetry reasons $P_{\Lambda}=0$ at $\eta=0$ in $p p$ collisions in the center of mass frame, hence the degree of transverse polarization $P_{\Lambda}$ around midrapidity is expected to be very small. As an alternative to $p+p \rightarrow \Lambda^{\uparrow}+X$, it has been suggested [4] to perform $\Lambda$ polarization studies in the process $p+p \rightarrow\left(\Lambda^{\uparrow}\right.$ jet $)+$ jet $+X$, where the $\Lambda$ and jets can be measured in the midrapidity region without paying a suppression penalty. It is especially of interest at LHC, where the factorized description is expected to apply and certain simplifications may arise due to the large center of mass energy $\sqrt{s}$.

\section{Spontaneous $\Lambda$ polarization in a factorized approach}

The magnitude of the $\Lambda$ polarization in $p+p \rightarrow \Lambda^{\uparrow}+X$ at large $x_{F}$ remains large up to the highest measured transverse momentum $p_{T}$ of the $\Lambda: p_{T} \sim 4 \mathrm{GeV} / c$. For sufficiently high $p_{T}$, perturbative QCD and collinear factorization should become applicable. Consider for example the $q g \rightarrow q g$ subprocess contribution to the $p+p \rightarrow \Lambda+X$ cross section in collinear factorization. It is of the form: $\sigma \sim q\left(x_{1}\right) \otimes g\left(x_{2}\right) \otimes \hat{\sigma}_{q g \rightarrow q g} \otimes D_{\Lambda / q}(z)$, where $q\left(x_{1}\right)$ is the quark density in proton $1, g\left(x_{2}\right)$ is the gluon density in proton 2 , and $D_{\Lambda / q}(z)$ is the $q \rightarrow \Lambda$ fragmentation function (FF). In a similar way the transverse polarization should be of the form: $P_{\Lambda} \sim q\left(x_{1}\right) \otimes g\left(x_{2}\right) \otimes \hat{\sigma}_{q g \rightarrow q g} \otimes$ ?, involving the unpolarized parton densities and the unpolarized hard partonic subprocess because $\Lambda$ polarization created in the hard partonic scattering is very small, $P_{\Lambda} \sim \alpha_{s} m_{q} / \sqrt{\hat{s}}$ [5]. The question mark indicates that for symmetry reasons at leading twist there is no collinear fragmentation function describing $q \rightarrow \Lambda^{\uparrow} X$. In collinear factorization $P_{\Lambda}$ is thus necessarily power suppressed. Dropping the demand of collinear factorization, does allow for a leading twist solution: the transverse momentum dependent (TMD) fragmentation function $D_{1 T}^{\perp}\left(z, \mathbf{k}_{T}\right)$ [6] for $\Lambda$ 's. It describes a nonperturbative $\mathbf{k}_{T} \times \mathbf{S}_{T}$ dependence in the fragmentation process, which is allowed by the symmetries (parity and time reversal). As the $\Lambda$ polarization arises in the fragmentation of an unpolarized quark, it carries the descriptive name "polarizing fragmentation function" [7]. $D_{1 T}^{\perp}$ has been extracted [7] from the low energy $p+p / B e \rightarrow \Lambda^{\uparrow} / \bar{\Lambda}^{\uparrow}+X$ data, where gluon FFs $(g \rightarrow \Lambda X)$ are expected to be hardly relevant. Reasonable valence quark FFs are obtained: $D_{1 T}^{\perp}$ has opposite signs for $u / d$ versus $s$ quarks, and the latter is larger. This is responsible for the cancellations that ensure $P_{\bar{\Lambda}} \approx 0$. The extraction has been done under the restriction of $p_{T}>1 \mathrm{GeV} / c$, in order to exclude the soft regime but to retain sufficient data to make a fit to. Whether this restriction is sufficiently

\footnotetext{
${ }^{1}$ An alternative may be to use neutral decays $\Lambda \rightarrow n \pi^{0}$ (50\% less frequent than $p \pi^{-}$) [3].
} 
strict is a matter of concern, due to the large $K$ factors required to obtain a cross section description. Data at higher $\sqrt{s}$ and $p_{T}$ would be much safer to ensure the validity of the factorized description. As pointed out, these do not necessarily require large $x_{F}$, if one goes beyond $p+p \rightarrow \Lambda^{\uparrow}+X$. If the origin of the transverse $\Lambda$ polarization is indeed due to polarizing fragmentation, then another, related asymmetry could be observed that does not need to vanish at $\eta_{\Lambda}=0$, namely in the process $p+p \rightarrow\left(\Lambda^{\uparrow}\right.$ jet $)+$ jet $+X[4]$ and actually also in $p+p \rightarrow\left(\Lambda^{\uparrow}\right.$ jet $)+X$.

\section{Jet- $\Lambda^{\uparrow}$ production}

The suggestion of [4] is to select two-jet events and to measure the jet momenta $K_{j}$ and $K_{j^{\prime}}$ (with $K_{j} \cdot K_{j^{\prime}}=\mathscr{O}(\hat{s})$ ), in addition to the momentum $K_{\Lambda}$ and polarization $S_{\Lambda}$ of the $\Lambda$ that is part of either of the two jets. A single spin asymmetry proportional to $\varepsilon_{\mu v \alpha \beta} K_{j}^{\mu} K_{j^{\prime}}^{v} K_{\Lambda}^{\alpha} S_{\Lambda}^{\beta}$ can then arise, which is neither power suppressed, nor needs to be zero at midrapidity. In the center of mass (c.o.m.) frame of the two jets the asymmetry is of the form:

$$
\mathrm{SSA}=\frac{d \sigma\left(+\mathbf{S}_{\Lambda}\right)-d \sigma\left(-\mathbf{S}_{\Lambda}\right)}{d \sigma\left(+\mathbf{S}_{\Lambda}\right)+d \sigma\left(-\mathbf{S}_{\Lambda}\right)}=\frac{\hat{\mathbf{K}}_{j} \cdot\left(\mathbf{K}_{\Lambda} \times \mathbf{S}_{\Lambda}\right)}{z M_{\Lambda}} \frac{d \sigma_{T}}{d \sigma_{U}}
$$

The analyzing power $d \sigma_{T} / d \sigma_{U}$ of the asymmetry depends on $D_{1 T}^{\perp}$. This new $\Lambda+$ jets observable could allow for a more trustworthy extraction of $D_{1 T}^{\perp}$ (for both quarks and gluons) and subsequent predictions, for instance for semi-inclusive DIS [8].

At RHIC and LHC this process $p p \rightarrow\left(\Lambda^{\uparrow}\right.$ jet $)$ jet $X$ can be studied. For instance, the ALICE experiment has excellent PID capabilities which allow measurements of $\Lambda$ 's over a wide $p_{T}$ range. The ALICE rapidity coverage is $-0.9 \leq \eta \leq+0.9$. If the jet rapidities $\left(\eta_{j, j^{\prime}}\right)$ are in this range and if gluon fragmentation is at least as important as quark fragmentation for both unpolarized and polarized $\Lambda$ production, then the process is dominated by gluon-gluon $(g g \rightarrow g g)$ scattering $^{2}$, i.e. $d \sigma_{T} / d \sigma_{U} \approx D_{1 T}^{\perp g}\left(z, K_{\Lambda T}^{2}\right) / D_{1}^{g}\left(z, K_{\Lambda T}^{2}\right)$. Because no model or fit for $D_{1 T}^{\perp g}$ is available yet, no predictions can be made in this case. If it happens that $D_{1 T}^{\perp g} \ll D_{1 T}^{\perp q}$, then one can use the extracted $D_{1 T}^{\perp q}$ to obtain an estimate. Taking into account the $q g \rightarrow q g$ subprocess, one finds for $\eta_{j^{\prime}} \approx-\eta_{j} \approx 0$

$$
\frac{d \sigma_{T}}{d \sigma_{U}} \approx \frac{\sum_{q} f_{1}^{q}(x) D_{1 T}^{\perp q}\left(z, K_{\Lambda T}^{2}\right)}{\sum_{q} f_{1}^{q}(x)\left(D_{1}^{q}\left(z, K_{\Lambda T}^{2}\right)+D_{1}^{g}\left(z, K_{\Lambda T}^{2}\right)\right)+f_{1}^{g}(x) D_{1}^{g}\left(z, K_{\Lambda T}^{2}\right) / 0.4},
$$

where $x \approx 2\left|\mathbf{K}_{j \perp}\right| / \sqrt{s}$. The factor 0.4 comes from the ratio $d \hat{\sigma}_{q g \rightarrow q g} / d \hat{\sigma}_{g g \rightarrow g g}$ at midrapidity; other partonic channels can be neglected [4]. For estimates we will use the DSV [10] and IMR [11] unpolarized FFs sets, and their accompanying $D_{1 T}^{\perp}$ parametrizations from Ref. [8]. Both DSV and IMR satisfy $D_{1}^{g} \ll D_{1}^{q}$ at larger $z$, as both have been obtained from fits to $e^{+} e^{-} \rightarrow \Lambda X$ data only, which is not sensitive to the fragmentation process $g \rightarrow \Lambda X$. The resulting asymmetry (3.2) evaluations are given in Fig. 1 for three different values of the $\Lambda$ momentum component transverse to the jet direction (see [12] for additional comments). Very small asymmetries are obtained at smaller $z$, due to $D_{1 T}^{\perp}$ being fitted to low energy data where mostly valence quarks matter. This behavior need not be realistic at high energies. ALICE would have most data in the region $z<0.5$ and could therefore provide valuable information on sea quark and gluonic contributions.

\footnotetext{
${ }^{2}$ Unlike in Ref. [4], here it will be assumed that universality of $D_{1 T}^{\perp}$ holds throughout [9].
} 

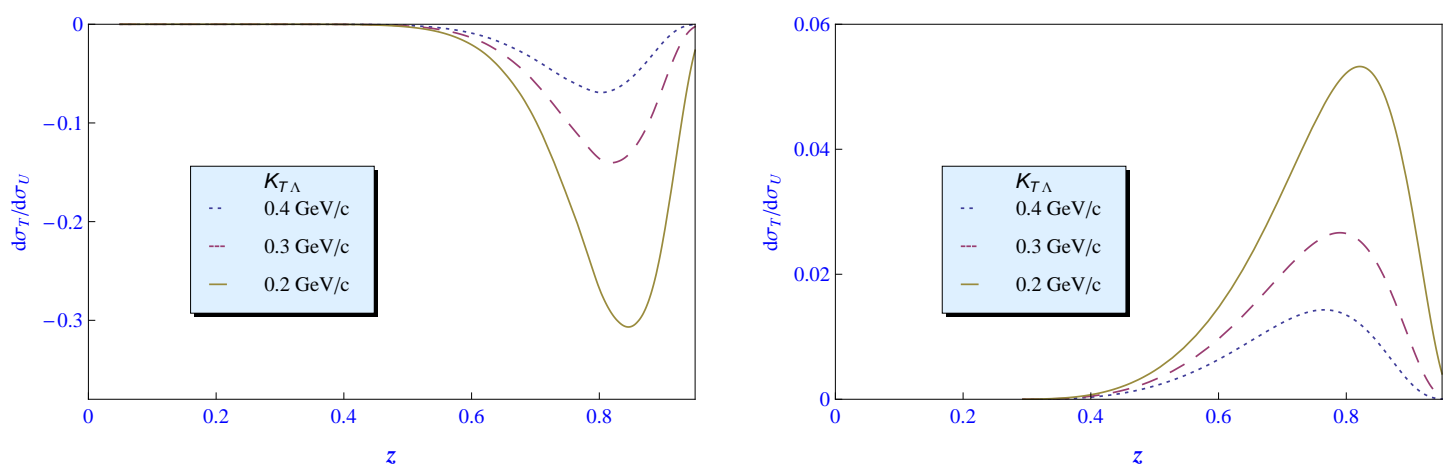

Figure 1: The asymmetry $d \sigma_{T} / d \sigma_{U}$ for $\eta_{j}, \eta_{j^{\prime}}=0,\left|K_{j \perp}\right|=\left|K_{j^{\prime} \perp}\right|=70 \mathrm{GeV}, \sqrt{s}=14 \mathrm{TeV}$, using DSV (left) and IMR (right) FFs.

The asymmetry is quite sensitive to the cancellation between $u / d$ and $s$ contributions, like in SIDIS [8], and can even flip its overall sign depending on the amount of $S U$ (3) breaking in the unpolarized fragmentation functions: IMR includes $S U(3)$ breaking, whereas DSV does not. This aspect represents a large uncertainty, but more reliable estimates are not possible at this stage. ALICE may help to determine the importance of $S U(3)$ breaking and flavor cancellations.

Unpolarized $\Lambda$ FFs have also been obtained taking into account midrapidity hadronic $\Lambda$ production data from STAR at RHIC: the AKK [13] and AKK08 [14] sets. These have very different characteristics compared to fits to $e^{+} e^{-}$data only. Fig. 2 shows the ratio $D_{1}^{g} / D_{1}^{q+\bar{q}}$ for $q=u, d, s$, respectively, for DSV, AKK and AKK08. The curves differ widely and represent yet another uncertainty in the predictions of (3.2). No $D_{1 T}^{\perp}$ extraction has been performed with AKK or AKK08. A problem with that is that the most recent fit AKK08 considerably undershoots the cross section of $p p \rightarrow \Lambda / \Lambda X$ at midrapidity, even at $\sqrt{s}=200 \mathrm{GeV}$ and transverse momenta in the range $2 \leq \mathbf{p}_{T} \leq 5 \mathrm{GeV} / c$. In [14] it was therefore concluded that there is "a possible inconsistency between the $p p$ and $e^{+} e^{-}$reaction data for $\Lambda / \bar{\Lambda}$ production" (cf. Fig. 5 of [14]). This issue ought to be clarified first.
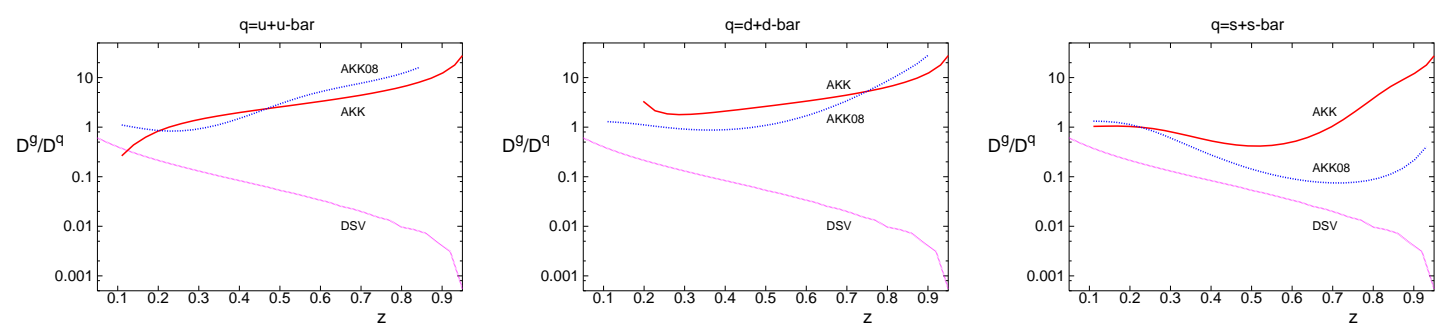

Figure 2: The ratio $D_{1}^{g} / D_{1}^{q+\bar{q}}$ as function of $z$ for $q=u, d, s$, respectively, for the leading order DSV, AKK and AKK08 sets, at the scale $Q=10 \mathrm{GeV}$.

The suggestion of [4] requires the opposite side jet $j^{\prime}$ to be measured, such that the jet-jet or equivalently the partonic c.o.m. frame can be selected. However, if universality of $D_{1 T}^{\perp}$ and gauge invariance of the factorized expression are taken to hold, then one can actually consider $p p \rightarrow$ $\left(\Lambda^{\uparrow}\right.$ jet $) X$, increasing the statistics considerably. The asymmetry is also given as in Eq. (3.1), but now in the hadronic c.o.m. frame, which can be shown by considering the Sudakov decomposition 
of the fragmenting quark momentum $k=K_{\Lambda} / z+\sigma_{\Lambda} n_{\Lambda}+k_{T}$ (but not of the other momenta), with the uncommon choice $n_{\Lambda}=P_{1}+P_{2}$. Due to the large $\sqrt{s}$ at the LHC, $\sigma_{\Lambda}$ is still suitably suppressed (at least by a factor $\left.E_{j} / \sqrt{s}\right)$, despite $n_{\Lambda}$ being non-lightlike. The asymmetry is now $\propto \varepsilon_{\mu v \alpha \beta} K_{j}^{\mu}\left(P_{1}+\right.$ $\left.P_{2}\right)^{v} K_{\Lambda}^{\alpha} S_{\Lambda}^{\beta}$, which in the hadronic c.o.m. frame indeed becomes $\propto \mathbf{K}_{j} \cdot\left(\mathbf{K}_{\Lambda} \times \mathbf{S}_{\Lambda}\right)$. Eq. (3.2) is now replaced by

$$
\frac{d \sigma_{T}}{d \sigma_{U}} \approx \frac{\int \frac{d y}{y} \sum_{q}\left(f^{q g}+f^{g q}\right) d \hat{\sigma}_{q g} D_{1 T}^{\perp q}}{\int \frac{d y}{y}\left[\sum_{q}\left(f^{q g}+f^{g q}\right) d \hat{\sigma}_{q g}\left(D_{1}^{q}+D_{1}^{g}\right)+f^{g g} d \hat{\sigma}_{g g} D_{1}^{g}\right]},
$$

where the arguments of the fragmentation functions have been suppressed and we introduced the notation: $f^{a b} \equiv x_{1} f_{1}^{a}\left(x_{1}\right) x_{2} f_{1}^{b}\left(x_{2}\right), d \hat{\sigma}_{a b} \equiv d \hat{\sigma}_{a b \rightarrow a b}(y)+d \hat{\sigma}_{a b \rightarrow a b}(1-y)$ with $y=-\hat{t} / \hat{s}$. The momentum fractions are fixed to be $x_{1}=x_{\perp} e^{\eta} /(2(1-y))$ and $x_{2}=x_{\perp} e^{-\eta} /(2 y)$ for given observed $x_{\perp}=2\left|\mathbf{K}_{j \perp}\right| / \sqrt{s}$ and $\eta=\eta_{j}$. The $y$ integration is between $y_{\min }=x_{\perp} e^{-\eta} / 2$ and $y_{\max }=1-x_{\perp} e^{\eta} / 2$. The expressions for the partonic cross sections can be found in Ref. [4]. For $D_{1}^{g} \gg D_{1}^{q}$ the last term in the denominator is the dominant one: for $x_{\perp}=0.01, \eta=0$ it is approximately a factor of 3 larger than the other term proportional to $D_{1}^{g}$.

\section{Acknowledgments}

I thank Markus Diehl, Dae Sung Hwang, Piet Mulders, Pasquale Di Nezza, Werner Vogelsang, and Feng Yuan for useful discussions.

\section{References}

[1] A. Lesnik et al., Phys. Rev. Lett. 35 (1975) 770;

G. Bunce et al., Phys. Rev. Lett. 36 (1976) 1113.

[2] A. D. Panagiotou, Int. J. Mod. Phys. A5 (1990) 1197.

[3] B. Cork et al., Phys. Rev. 120 (1960) 1000;

S. Olsen et al., Phys. Rev. Lett. 24 (1970) 843.

[4] D. Boer, C. J. Bomhof, D. S. Hwang and P. J. Mulders, Phys. Lett. B659 (2008) 127.

[5] G. L. Kane, J. Pumplin and W. Repko, Phys. Rev. Lett. 41 (1978) 1689.

[6] P. J. Mulders and R. D. Tangerman, Nucl. Phys. B461 (1996) 197.

[7] M. Anselmino, D. Boer, U. D’Alesio and F. Murgia, Phys. Rev. D63 (2001) 054029.

[8] M. Anselmino, D. Boer, U. D’Alesio and F. Murgia, Phys. Rev. D65 (2002) 114014.

[9] A. Metz, Phys. Lett. B549 (2002) 139;

L. P. Gamberg, A. Mukherjee and P. J. Mulders, Phys. Rev. D77 (2008) 114026;

S. Meissner and A. Metz, Phys. Rev. Lett. 102 (2009) 172003;

F. Yuan and J. Zhou, Phys. Rev. Lett. 103 (2009) 052001.

[10] D. de Florian, M. Stratmann and W. Vogelsang, Phys. Rev. D57 (1998) 5811.

[11] D. Indumathi, H. S. Mani and A. Rastogi, Phys. Rev. D 58 (1998) 094014.

[12] D. Boer, arXiv:0907.1610 [hep-ph].

[13] S. Albino, B. A. Kniehl and G. Kramer, Nucl. Phys. B 734 (2006) 50.

[14] S. Albino, B. A. Kniehl and G. Kramer, Phys. Rev. Lett. 100 (2008) 192002. 